\title{
Effects of outer top gas injection on ICRF coupling in ASDEX Upgrade: towards modelling of ITER gas injection
}

\author{
W. Zhang ${ }^{1,2,3, a)}$, V. Bobkov ${ }^{2}$, J-M. Noterdaeme ${ }^{1,2}$, W. Tierens ${ }^{2}$, R. Bilato ${ }^{2}$, D. Carralero ${ }^{2}$, D. Coster ${ }^{2}$, \\ J. Jacquot ${ }^{2}$, P. Jacquet ${ }^{4}$, T. Lunt ${ }^{2}$, R.A. Pitts ${ }^{5}$, V. Rohde ${ }^{2}$, G. Siegl ${ }^{2}$, H. Fuenfgelder ${ }^{2}$, D. Aguiam ${ }^{6}$,
} A. Silva ${ }^{6}$, L. Colas ${ }^{7}$, S. Ceccuzzi ${ }^{8}$, the ASDEX Upgrade Team ${ }^{2}$

\author{
${ }^{1}$ Applied Physics Department, University of Ghent, Ghent, Belgium \\ ${ }^{2}$ Max-Planck-Institut für Plasmaphysik, Garching, Germany \\ ${ }^{3}$ Institute of Plasma Physics, Chinese Academy of Sciences, Hefei, P. R. China \\ ${ }^{4}$ CCFE, Culham Science Centre, Abingdon, UK \\ 5ITER Organization, Route de Vinon-sur-Verdon, CS 90 046, 13067 St. Paul Lez Durance Cedex, France \\ ${ }^{6}$ Instituto de Plasmas e Fusão Nuclear, IST, Universidade de Lisboa, Lisboa, Portugal \\ ${ }^{7}$ CEA, IRFM, F-13108 Saint-Paul-Lez-Durance, France \\ ${ }^{8}$ ENEA, Fusion Physics Division, Frascati, Italy \\ ${ }^{a)}$ Corresponding author: wei.zhang@ipp.mpg.de
}

\begin{abstract}
The influence of outer top gas injection on the SOL density and ICRF coupling has been studied in ASDEX Upgrade (AUG) L-mode plasmas for the first time. The 3D edge plasma fluid and neutral transport code EMC3-EIRENE is used to simulate the Scrape-Off Layer (SOL) plasma density, and the 3D wave code RAPLICASOL is used to compute the ICRF coupling resistance with the calculated density. Improvements have been made in the EMC3-EIRENE simulations by fitting transport parameters separately for each gas puffing case. It is found that the calculated local density profiles and coupling resistances are in good agreement with the experimental ones. The results indicate that the SOL density increase depends sensitively on the spreading of the injected outer top gas. If more gas enters into the main chamber through the paths near the top of vessel, the SOL density increase will be more toroidally uniform; if more gas chooses the paths closer to the mid-plane, then the SOL density increase will be more local and more significant. Among the various local gas puffing methods, the mid-plane gas valve close to the antenna is still the best option in terms of improving ICRF coupling. Differences between the outer top gas puffing in AUG and the outer top gas puffing in ITER are briefly summarized. Instructive suggestions for ITER and future plans for ITER gas injection simulations are discussed.
\end{abstract}

\section{Introduction}

Plasma heating with waves in the lon Cyclotron Range of Frequency (ICRF) relies on the fast wave to transfer the energy from the antenna straps to the plasma. However, an evanescent layer often exists in front of the ICRF antennas where the plasma density is below the fast wave cut-off density, usually on the order of $10^{18} \mathrm{~m}^{-3}$. In this evanescent layer the fast wave decays exponentially. To improve the ICRF coupling, the width of the layer must be reduced. One of the ways to do this is to increase the plasma density in this layer. Previous experiments and simulations in ASDEX Upgrade (AUG) [1-5] and in JET [6-8] indicate that by simply shifting the gas source from the lower divertor to the main chamber (top or mid-plane), the Scrape-Off Layer (SOL) density and thus the ICRF coupling can be greatly enhanced. 
To understand the influence of outer top gas injection on the SOL density and ICRF coupling, a series of L-mode discharges with various gas puffing locations, in particular from the upper part of the lower field side cross-section (outer top gas puffing), have been carried out in AUG. The three-dimensional (3D) edge plasma fluid and neutral transport code EMC3-EIRENE [9] is used to simulate the SOL density during different gas puffing. Effects of the ICRF waves on the SOL, such as the RF-sheath-driven plasma convection [10] and the neutral ionization by the near and far electric fields [11] are not considered in the EMC3-EIRENE simulations. The 3D wave code RAPLICASOL [12] is then used to calculate the coupling resistance with the 3D plasma density from the EMC3-EIRENE simulations. Compared to the previous 3D simulations of local gas puffing in H-mode plasmas in AUG (see [4]), the study in this paper has the following improvements:

1) Further investigations of local gas puffing in L-mode plasmas;

2) Outer top gas puffing, previously never examined, has been extensively studied.

3) Improved EMC3-EIRENE simulations with different settings of plasma transport parameters for different gas puffing cases.

4) The 3D wave code RAPLICASOL is used to calculate the coupling resistance for the first time.

It is worth mentioning that in ITER main chamber gas puffing will be performed through injection pipes routed behind the blanket modules and located just below the upper port plugs near the upper part of the chamber on the low field side [6]. The gas injection locations are similar to those of the outer top gas puffing in AUG. However, in ITER, the injection outlets must be located behind the first wall panels to avoid neutronic heating of the pipes. The result is that gas disperses through multiple gaps between the blanket modules and is not poloidally localized. The gas spreading thus differs between ITER and AUG. Nevertheless, some of the conclusions inferred from gas puffing studies in AUG may also be applicable to ITER.

The outline of the paper is as follows. In Section 2, experimental and simulation configurations of the AUG outer top gas puffing are discussed. The fitting of transport coefficients in the EMC3-EIRENE simulations are discussed in detail. In Section 3, the calculated density profile and coupling resistance of ICRF antennas are compared with the experimental values for different gas puffing cases. The mechanisms leading to an increase in the SOL density during local gas puffing are explained using field line tracing. In Section 4, the influence of outer top gas valve locations on the SOL density are discussed. Finally, Section 5 provides conclusions and outlook.

\section{Experimental and simulation configurations}

\subsection{Outer top gas puffing in AUG}

The various gas puffing locations available in AUG, including the lower divertor (DIV), the inner top (TOPFO2), the outer top (TOPCO2) and the mid-plane (MID) are illustrated in figure 1. For divertor gas fueling, four evenly distributed gas valves in the lower divertor are switched on simultaneously. The mid-plane gas valve is located very deep in the A-port and produces a homogenous gas cloud which fills the whole A-port [4]. Injection from the inner top valve has been the subject of a detailed earlier study [4] and is not considered further here. The injection and approximate spreading of gas from the TOPCO2 location are shown in figure 2. Gas injected from the TOPCO2 location enters via a long port. Part of the gas enters the main chamber directly through gaps in the outer top of 
vessel (path A) and part spreads downward, reaching the plasma through A-ports in the mid-plane (path B), since the ICRF antennas are almost impenetrable. This blocked pathway for the gas is considered in the EMC3-EIRENE simulations by setting wall structures at the locations of ICRF antennas.

In the simulations, cases (Table 1) with different combinations of gas paths are investigated: both paths $A$ and $B$ are open (Case II); only path A (Case III) or only path B (Case IV) is open. Case II is expected to be closest to the real experimental situation. These three cases are mainly to understand the influences of gas paths on the SOL density. The DIV (Case I), the MID3 (Case V) and the MID13 (Case VI) gas puffing cases are used for comparison with the TOPCO2 simulations. More details of the simulated cases and the corresponding experiments are given in Table 1.

\begin{tabular}{|l|l|l|l|}
\hline Simulated cases & $\begin{array}{l}\text { Case } \\
\text { number }\end{array}$ & $\begin{array}{l}\text { Gas valve positions } \\
\text { (toroidal) }\end{array}$ & $\begin{array}{l}\text { Corresponding } \\
\text { experiments }\end{array}$ \\
\hline Lower divertor gas (DIV) & Case I & $\begin{array}{l}\text { Sector } 1\left(-168.75^{\circ}\right) \\
\text { Sector } 5\left(-78.25^{\circ}\right) \\
\text { Sector } 9\left(11.25^{\circ}\right) \\
\text { Sector } 13\left(101.25^{\circ}\right)\end{array}$ & $\begin{array}{l}\# 33262(1.40-2.75 \mathrm{~s}) \\
\# 33265(2.80-5.16 \mathrm{~s})\end{array}$ \\
\hline TOPCO2 gas, path A + path B & Case II & Sector 2 $\left(\Phi_{\mathrm{v}}=-146.25^{\circ}\right)$ & $\# 33264(1.40-2.80 \mathrm{~s})$ \\
\hline TOPCO2 gas, path A & Case III & Sector 2 $\left(\Phi_{\mathrm{v}}=-146.25^{\circ}\right)$ & \\
\hline TOPCO2 gas, path B & Case IV & Sector 2 $\left(\Phi_{\mathrm{v}}=-146.25^{\circ}\right)$ & \\
\hline Sector 3 mid-plane gas, (MID3) & Case V & Sector $3\left(\Phi_{\mathrm{v}}=-123.75^{\circ}\right)$ & $\# 33264(2.80-5.32 \mathrm{~s})$ \\
\hline Sector 13 mid-plane gas, (MID13) & Case VI & Sector $13\left(\Phi_{\mathrm{v}}=101.25^{\circ}\right)$ & $\# 33263(1.40-2.75 \mathrm{~s})$ \\
\hline
\end{tabular}

Table 1. The toroidal positions of gas valves and the corresponding experiments. The simulated TOPCO2 gas puffing cases (Cases II, III and IV) differ only in terms of the selected gas pathways. In all simulations, the same gas puffing rate of $6.5 \times 10^{21}$ electrons $/ \mathrm{s}$ is used.

The toroidal locations of the ICRF antennas and density diagnostics in the 2016 experimental campaign are shown in figure 1(b). Antennas 1 and 3 are boron-coated 2-strap antennas, whereas antennas 2 and 4 are tungsten-coated 3-strap antennas. More details of the current state of the ICRF antennas can be found in [13]. For the experiments in this paper, Antennas 1 and 3 or Antennas 2 and 4 are powered in pairs. The ICRF heating scheme is hydrogen minority in deuterium ( $f_{\text {ICRF }}=36.5 \mathrm{MHz}$ ), with a plasma current of $I_{p}=0.8 \mathrm{MA}$ and toroidal magnetic field of $B_{T}=2.5 T$. The Lithium beam (Li-Beam) diagnostic is used to determine the transport coefficients in the simulations and is located far away from the TOPCO2 and mid-plane (including MID3 and MID13) gas valves. The antenna reflectometer diagnostics (embedded in Antenna 4) are close to the MID13 gas valve. They are used for comparisons with the simulated local density profiles in each gas puffing case.

\subsection{Simulation setup and fitting of transport coefficients}

EMC3-EIRENE is a code package which couples the EMC3 [9] and EIRENE [14] codes self-consistently in 3D geometry. EMC3 solves a set of time-independent fluid equations for the mass, momentum, electron and ion energies. EIRENE computes the kinetic Boltzmann equations and handle all the atomic physics. More details of the code can be found in [9].

In the simulations, toroidal axisymmetry cannot be assumed owing to the toroidally inhomogeneous SOL density during local gas puffing. Thus, we have built a toroidal $360^{\circ}$ computational grid based on the magnetic equilibrium reconstruction from AUG pulse \#33262 at 2.30s (figure 3). The grid is composed of 16 equally 
constructed segments with each divided into the core, SOL and Private Flux Region (PFR) zones. Good spatial resolution is chosen in the SOL in order to guarantee the accuracy of neutral transport and ionization there. To make the simulations more realistic, plasma-facing components such as the divertor, the limiters, the top and inner wall structures are included in the simulation model. No gas pump is set in the simulations since the vast majority of gas is pumped out homogenously by the toroidally axisymmetric cryo-pump. The gas puffing is set by treating a small part of the recycling flux as the gas puffed from the valve positions. In an L-mode plasma (e.g. \#33262), an experimental gas puff rate of $\Phi_{D 2}=6.5 \times 10^{21} \mathrm{el} / \mathrm{s}$ is equal to $\sim 10 \%$ of the total recycling flux in the simulations. However in a H-mode plasma (e.g. \#31269), an experimental gas puff rate of $\Phi_{D 2}=1.2 \times 10^{22} \mathrm{el} / \mathrm{s}$ is only $6 \%$ of the total recycling flux [4].

The parallel transport of plasma in EMC3-EIRENE is purely neoclassical while the perpendicular transport depends on the prescribed perpendicular transport parameters. The perpendicular transport coefficients for particles and heat $\left(D_{\perp}\right.$ and $\left.\chi_{\perp}\right)$ in the simulations are chosen by using data from various diagnostics such that the computed upstream and downstream profiles are best matched to the experiment. Examples of time traces of main plasma parameters during DIV, TOPCO2 and MID13 gas puffing are given in figure 4 . In these experiments, $I_{p}$ and the total heating power $0.81 \mathrm{MW}$ are fixed. The gas puff rate during DIV gas puffing $\left(\Phi_{D 2}=7.5 \times 10^{21} \mathrm{el} / \mathrm{s}\right)$ is larger than that during MID13 or TOPCO2 gas puffing $\left(\Phi_{D 2}=6.5 \times 10^{21} \mathrm{el} / \mathrm{s}\right)$. However, the SOL density is lower for DIV puffing leading to a suspicion that a small part of the gas puffed from the divertor has been directly pumped out before being fully ionized, leading to a lower gas fueling efficiency and thus lower global density. Since the plasma densities obtained for the 3 gas puffing locations are different, the simulated plasmas for the DIV (\#33262 at 2.3s), TOPCO2 (paths A+B, \#33262 at 3.8s) and MID13 (\#33263 at 2.3s) cases must be generated separately. This is different from previous EMC3-EIRENE simulations in H-mode (see $[4,7])$ in which the global density is almost unchanged as the gas puffing location is varied so that the same background plasma could be used with the gas valve location as the only variable.

Radial profiles of SOL transport coefficients obtained from the fitting processes are shown in figure 5, along with some key experimental profiles and their comparison with the simulations. Good agreement is found between experimental and simulated profiles. Agreement is found between experimental and simulated profiles. In the simulations, the upstream profiles (mid-plane density and temperature) are best fitted to the experimental measurements because the SOL density in front of the antennas and the electron temperature near the gas valves (associated with ionization) are important parameters in our study. Discrepancies between model and experiment $\mathrm{J}_{\text {sat }}$ values (such as those in the TOPCO2 (\#33262 @ 3.8s) puffing case) are expected to have much less effects on the final density profiles in front of the antennas and the coupling resistance. On the other hand, the relative change of coupling resistance is much less sensitive to the changes in transport parameters.

It is worth mentioning that although we tried our best to fit the simulated mid-plane temperature profiles to the Langmuir probe data, some discrepancies can still be seen in the near SOL. Given fixed input power and specified upstream density profiles, the EMC3-EIRENE code is unable to generate temperature profiles which have very large gradients in the near SOL as shown by the measurements, no matter what kind of energy transport parameter profiles are used. The reason for this is still unclear. It is suspected that the lack of drifts in the simulations may attribute to this. On the other hand, the experimental error bars relax the discrepancies when fitting the profiles. Having obtained the 3D densities for the different gas puffing cases from EMC3-EIRENE, the 3D wave coupling code RAPLICASOL is used to calculate the coupling resistances which can then be compared with their experimental values (see section 3.2). 


\section{Simulation results}

\subsection{Influence of outer top gas puffing on the SOL plasma}

Figure 6 shows the simulated neutral density $\left(n_{D 2}+n_{D}\right.$, i.e. neutral molecular density + neutral atom density) and electron density $\left(n_{e}\right)$ during TOPCO2 and MID3 gas puffing. In the case of TOPCO2 gas puffing, four equidistant point sources in the outer top of the vessel have been used in the simulations to replicate the experimental gas injection. The mid-plane gas valve is set at the outer edge $(\mathrm{Z}=0.0 \mathrm{~m})$ of the computational grid to generate a gas cloud which has the same poloidal and toroidal extent as that seen in experiment with visible cameras.

The simulated neutral densities indicate that different selections of gas paths result in substantially different spreading and intensity of the neutral gas. When both paths A and B are open (Case II), about 53\% of the TOPCO2 gas follows path $A$ with the rest following path $B$. Two gas clouds are developed inside the vessel: the outer midplane cloud has larger spatial extent but lower neutral density than that at the outer top location. When only path A is open (Case III), a localized gas cloud with high neutral density is formed in the outer top of vessel. The low neutral density found at the outer mid-plane originates mainly from recycling fluxes generated at the limiters. When only path B is open (Case IV), all the gas has to reach its destination through the A-ports and an extended gas cloud is generated at the outer mid-plane. In this cloud, the neutral density decays vertically from $\mathrm{Z}=0.5 \mathrm{~m}$ to $\mathrm{Z}=-0.5 \mathrm{~m}$.

A neutral gas cloud often leads to a localized plasma electron density increase due to local ionization. As shown in figure 6, this occurs for both TOPCO2 (Cases II and III) and mid-plane gas puffing (Case V). To better understand the SOL electron density distributions, figure 7 provides toroidal cross-sections in the mid-plane. For TOPCO2 gas puffing, when both paths A and B are open (Case II), the SOL density increases almost toroidally uniformly. Slightly higher SOL densities are found in toroidal regions near Antenna 3 and the TOPCO2 gas valve, which are induced by the gas following paths $A$ and $B$, respectively. When only path $A$ is open (Case III), the SOL density is increased to about the same level as for Case II. A higher SOL density is found in toroidal regions near Antenna 3 (magnetically connected to the top gas cloud). When only path B is open (Case IV), a significant local density increase is found in toroidal regions near the TOPCO2 gas valve. This density increase decays gradually with increasing toroidal distance from the gas valve. The SOL density increase for Case IV is lower than for Case V (mid-plane gas puffing) because in the former, the gas cloud generated in the outer mid-plane has a much larger toroidal extent, but lower intensity than for the latter.

We have also compared the simulated densities with the antenna reflectometer measurements $\left(\Phi=73.83^{\circ}\right.$, $\mathrm{Z}=0.0 \mathrm{~m})$, as shown in figure 8 . The measured density profiles are averaged over the time intervals where only $\mathrm{ECRH}$ power is used, i.e. \#33262, $t=[2.02,2.40 \mathrm{~s}]$ for the DIV puffing; \#33262, $t=[3.52,3.90 \mathrm{~s}]$ for the TOPCO2 puffing; $\# 33263, t=[2.02,2.40 \mathrm{~s}]$ for the MID13 puffing; \#33264, $t=[3.52,3.90 \mathrm{~s}]$ for the MID3 puffing. During these periods, no RF convective cells are driven in front of antenna 4 and the measured density profiles are not influenced by the RF driven convection. It is known that the largest SOL density increase during mid-plane gas puffing is near the gas valve. Because the reflectometer is toroidally far away from the MID3 gas valve ( 6m), but close to the MID13 gas valve $(\sim 0.86 \mathrm{~m})$, the density values measured by the antenna reflectometer during the MID13 gas puffing are much larger. In contrast, although the TOPCO2 gas valve is far away from the reflectometer ( $\sim 2 \mathrm{~m})$, it increases the SOL density almost toroidal uniformly and at a moderate level. This explains why the measured density profile (at $\Phi=73.83^{\circ}$ ) during TOPCO2 gas puffing is even larger than that observed during MID3 gas puffing. In the simulations, the density profiles (at $\Phi=73.83^{\circ}$ ) of the TOPCO2 gas puffing cases (Cases II, III and IV) are almost the same in the far SOL. Good agreement is found between the simulated and measured electron density profiles for densities in 
the range $\left[0.2 \times 10^{19}, 0.6 \times 10^{19} \mathrm{~m}^{-3}\right]$. However, there are clearly discrepancies in the region towards the very edge. This is mainly due to the fact that the reflectometer can only reliably measure density profiles beginning at some distance in front the microwave horn antennas, while in the simulations the density profiles start at the hard limit of the wall.

Whether a local density cloud leads to a local or a global SOL density increase depends on the magnetic field topology. To examine this (see Figure 9), field line tracing has been performed for four points in the top (A1, $A 2$, $A 3, A 4)$ and four (B1, B2, B3, B4) in the mid-plane gas clouds. Field lines originating from the top towards the mid-plane spread over a large poloidal extent, whereas those tracked from the mid-plane towards the top or bottom are more concentrated. This explains the more localized density increase during mid-plane gas puffing and the more toroidally uniform density increase during top gas puffing. For TOPCO2 gas puffing (path $A+B$ ), the SOL density increase depends strongly on the quantity of gas which passes through each gas path. If more follows path A, the top gas cloud plays a more important role and the SOL density in regions near Antenna 3 will be larger; if more gas selects path $\mathrm{B}$, the mid-plane gas cloud dominates and the SOL density increase in front of Antennas 1 and 2 will be larger.

\subsection{Influence of the outer top gas on ICRF coupling}

Previous studies of RF wave coupling [4] used EM3C-EIRENE simulated density profiles as input to the FELICE code [15]. However, since FELICE is a 1D code, the 3D inhomogeneous density distribution tailored by local gas puffing must be averaged to $1 \mathrm{D}$, introducing errors in the calculated results. The coupling resistances $\left(R_{c}\right)$ computed with the combined EMC3-EIRENE and FELICE simulations can only be in qualitative agreement with the experimental values [4]. To handle the 3D density, the finite-element code RAPLICASOL (Radiofrequency wAve couPLing for lon Cyclotron Antenna in Scrape-Off-Layer) [12] has been used here to numerically calculate $\mathrm{R}_{\mathrm{c}}$. Given a 3D EMC3-EIRENE plasma density, RAPLICASOL solves Maxwell's equations in the cold plasma approximation in the frequency domain with realistic 3D antenna geometry. It does this for both ports of an antenna independently in order to compute the scattering matrix (S-matrix). The RAPLICASOL simulation domain is terminated on the plasma side by an absorbing boundary condition (PML) [16]. The effects of the core plasma are not taken into account.

In the RAPLICASOL calculations, a Feed Scheme ( $F S$, a vector with $N P=2$ elements, one for every port) is first determined such that the antenna has the desired total power output and the desired balance (both known from experiment). The total power is given by the sum of the power from all ports (denoted with subscript s)

$$
\begin{aligned}
& P_{\text {tot }}=\sum P_{s} \\
& P_{S}=\frac{1}{2} \frac{\left(F S_{s}\left(1+\left|S A C_{s}\right|\right)\right)^{2}}{Z_{0} S W R}
\end{aligned}
$$

where $S W R$ is the Standing Wave Ratio, $S A C_{s}$ is the port reflection coefficient for port $s$ and $\mathrm{Z}_{0}$ the input impedance:

$$
S W R_{S}=\frac{1+\left|S A C_{S}\right|}{1-\left|S A C_{s}\right|}
$$

$S A C_{s}$ is calculated in terms of the elements of the S-matrix $S_{i, j}$ : 


$$
S A C_{s}=\frac{\sum_{i=1}^{N P} S_{s, i} F S_{i}}{F S_{S}}
$$

The power balance is $P_{s} / P_{\text {tot }}$. These equations are solved for $F S$. The coupling resistance for port $s$ is then simply

$$
R_{c, s}=\frac{Z_{0}}{S W R_{S}}
$$

RAPLICASOL assumes the input impedance $Z_{0}=25 \Omega$. The $R_{c}$ value of an antenna is calculated by averaging the $\mathrm{R}_{c, s}$ values of the two ports. For 2-strap antennas, the left and right straps use one port each, with power balances of $\frac{1}{2}, \frac{1}{2}$, separately. For 3-strap antennas, the centre strap uses one port while the two outer straps share one port, with power balances of $\frac{2}{3}, \frac{1}{3}$, separately.

The experimental and the simulated Relative Change of Coupling Resistance (RCCR) are shown in figure 10. Here RCCR $=\Delta R_{c} / R_{c_{-} r e f}$, in which $\Delta R_{c}=R_{c}-R_{c_{-} r e f}$ and $R_{c_{-} r e f}$ is coupling resistance during divertor gas puffing and is used as a reference. For mid-plane gas puffing, the largest increase of $R_{c}$ is in the range of $60 \%-70 \%$ when the antenna is closest to the gas valve. This increase decays almost exponentially as the valve-antenna distance increases. The calculated RCCR values obtained with combined EMC3-EIRENE+RAPLICASOL simulations are in quantitative agreement with experiment, while the combined EMC3-EIRENE+FELICE predict only the correct trend, confirming the need to extend the coupling calculations to 3D when modelling the influence of gas injection location on coupling. For TOPCO2 gas puffing, the EMC3-EIRENE+RAPLICASOL calculations find that the RCCR values in Case II (when both Paths A and B are included) are lower than in Case IV (Path B only) when the valveantenna distance is lower than $4.2 \mathrm{~m}$. This is reasonable because in Case IV, all the gas enters the main chamber through the A-ports, leading to a local, but significant density and a consequent increase in $R_{c}$. In experiment, the TOPCO2 gas follows both Paths A and B so that the measured RCCR values should be in better agreement with Case II. However, since the measured RCCR values are in better agreement with Case IV, it can be inferred that the gas influx through Path B in experiments is much larger than assumed in the simulations for Case II. It is suspected that this is mainly because some detailed wall structures such as the toroidally discrete limiters at the top of vessel are not considered in the EMC3-EIRENE model. These wall structures will play a role in blocking the gas following Path A.

\section{Influence of top gas valve locations on SOL density}

To further understand the influence of positioning of the outer top gas valve on the SOL density, simulations have also been performed with gas valves located at different poloidal locations. The simulated background plasma from pulse \#33262 at 2.3s is used in all the cases, with the gas valve position the only variable. As might be expected, the region of locally enhanced density shifts in position as the gas injection location is displaced poloidally towards the mid-plane direction (figure 11). Since field lines penetrating these gas clouds are connected to different toroidal regions of the SOL, the SOL density in these cases is substantially different. The toroidal cross-sections of density in the mid-plane (figure 12) indicate that when the gas valve is closer to the top (Case A), the SOL density increase is more global but less significant; when the gas valve is closer to the mid-plane (Case D), the SOL density increase is more local and more substantial. This can be again explained by the field line tracing plots in figure 9: field lines originating from the top towards the mid-plane spread widely and influence the SOL density over a larger spatial extent but at a smaller level; field lines launched from the mid-plane are more 
concentrated and influence the SOL density over a smaller spatial region but to a much higher amplitude. The local distribution of the SOL density thus depends sensitively on the poloidal location of the gas valve position in the poloidal direction. To achieve a significant density increase in front of a given ICRF antenna, gas injection needs to occur as close as possible to the mid-plane, while in the toroidal direction the neutral cloud generated by gas puffing should be magnetically connected to the desired antennas.

\section{Conclusions}

This work is part of an effort to assess the effect of position of the ITER main chamber gas injection modules on the local SOL density in front of the ICRF antennas. We have studied the influence of gas injection location on improvements in the coupling resistance of ICRF on AUG L-mode plasmas, using the 3D code EMC3-EIRENE to simulate the SOL density distributions in the presence of different gas source in combination with the RF code RAPLICASOL to compute the antenna coupling resistance. In the EMC3-EIRENE simulations, the perpendicular plasma transport coefficients are determined separately for each gas puffing case by matching simulated SOL and divertor target profiles with experimental data.

The simulation results imply that in AUG, gas introduced from the top low field side of the poloidal cross-section enters the main chamber either through the gaps in the outer top port or through the mid-plane A-ports. The gas clouds generated in the outer top and outer mid-plane are the sources of increased density in the SOL. The simulations demonstrate that when both paths are open, the SOL density is increased almost toroidal uniformly. A slightly larger increase is found at toroidal positions near the gas valve and near the antenna, caused by the increased neutral gas densities in the mid-plane and at the top, respectively. When gas is allowed to penetrate only through the upper channel (i.e. without leakage of some gas towards the midplane) the SOL density increase is largely toroidally uniform, but a higher values are near antennas magnetically connected to the toroidally and poloidally localized injection point. When gas is allowed to follow a path directed towards the mid-plane, a significant, but local density increase is found at toroidal positions near the gas valve. This SOL density increase reduces with increasing toroidal separation from the valve.

The 3D code RAPLICASOL has been used to calculate the coupling resistance $\left(R_{c}\right)$ based on the 3D plasma density distributions obtained from the EMC3-EIRENE simulations. For mid-plane gas puffing, the calculated $R_{c}$ values are in quantitative agreement with those measured experimentally. The results indicate that the increase of $R_{c}$ is most significant for the antenna toroidally closest to the gas valve $(\sim 70 \%)$, and that this increase decays exponentially as the gas valve-antenna distance becomes larger. For gas puffing from the top low field side of the poloidal cross-section, the increase of $R_{c}$ is less significant (the largest increase is $\sim 40 \%-50 \%$ for antenna closest to the gas valve) and also depends exponentially on the valve-antenna distance. The measured $R_{c}$ values are in better agreement with the simulated values for the case in which the gas injected from the top location is assumed to penetrate behind structures down towards the outboard mid-plane.

A general and key conclusion, expected also to be applicable to ITER, can be drawn from the study reported in this paper: mid-plane gas puffing close to the antennas is most effective in improving ICRF coupling. However, ITER will employ gas injection which is routed behind blanket modules from injection pipes entering the main chamber through upper diagnostic ports. The injection lines must be hidden from the plasma since they cannot be sufficiently cooled to manage the neutronic heating. This means that injected gas will diffuse into the chamber through many gaps between blanket modules and is not expected to be a very localized source. Nevertheless, the

currently planned pipe locations mean that the peak in the poloidal neutral gas density distribution will occur 
somewhere just below the upper lateral ports. One of the main drivers for the selection of the toroidal locations of main chamber ports through which gas is to be injected on ITER was that a magnetic connection to the IRCF antennas exist for least one of the injection points for the baseline magnetic equilibrium $\left(q_{95}=3\right)$ and this choice is vindicated by the analysis presented in this paper. More detailed analysis is now required for ITER of the likely benefit to coupling resistance when a realistic gas entry distribution is included and full 3D modelling is applied. This paper has demonstrated that such analysis is meaningful and could be performed with reasonable confidence.

Acknowledgments: This work has been carried out within the framework of the EUROfusion Consortium and has received funding from the Euratom research and training programme 2014-2018 under grant agreement No 633053. The views and opinions expressed herein do not necessarily reflect those of the European Commission or of the ITER Organization.

\section{References}

[1] Jacquet P. et al 2012 Nuclear Fusion 52042002

[2] Bobkov V. et al 2014 AIP Conference Proceedings 1580271

[3] Zhang W. et al 2015 AIP Conference Proceedings 1689050006

[4] Zhang W. et al 2016 Nuclear Fusion 56036007

[5] Bobkov V. et al 2015 AIP Conference Proceedings 1689030004

[6] Jacquet P. et al 2016 Nuclear Fusion 56046001

[7] Zhang W. et al 2017 "3D simulations of gas puff effects on edge plasma and ICRF coupling in JET", Nuclear Fusion, accepted for publishing

[8] Lerche E. et al 2015 Journal of Nuclear Materials 463 634-9

[9] Feng Y. et al 2004 Contributions to Plasma Physics 44 57-69

[10] Zhang W. et al 2016 Plasma Phys. Control. Fusion 58095005

[11] Lyssoivan A. et al 2012 Plasma Physics and Controlled Fusion 54074014

[12] Jacquot J. et al 2015 Radiofrequency Power in Plasmas 1689050008

[13] Bobkov V. et al 2016 Nuclear Fusion 56084001

[14] Reiter D. et al 2005 Fusion Science and Technology 47 172-86

[15] Brambilla M. 1989 Plasma Physics and Controlled Fusion 31 723-57

[16] Berenger J. P. 1994 Journal of Computational Physics 114 185-200 

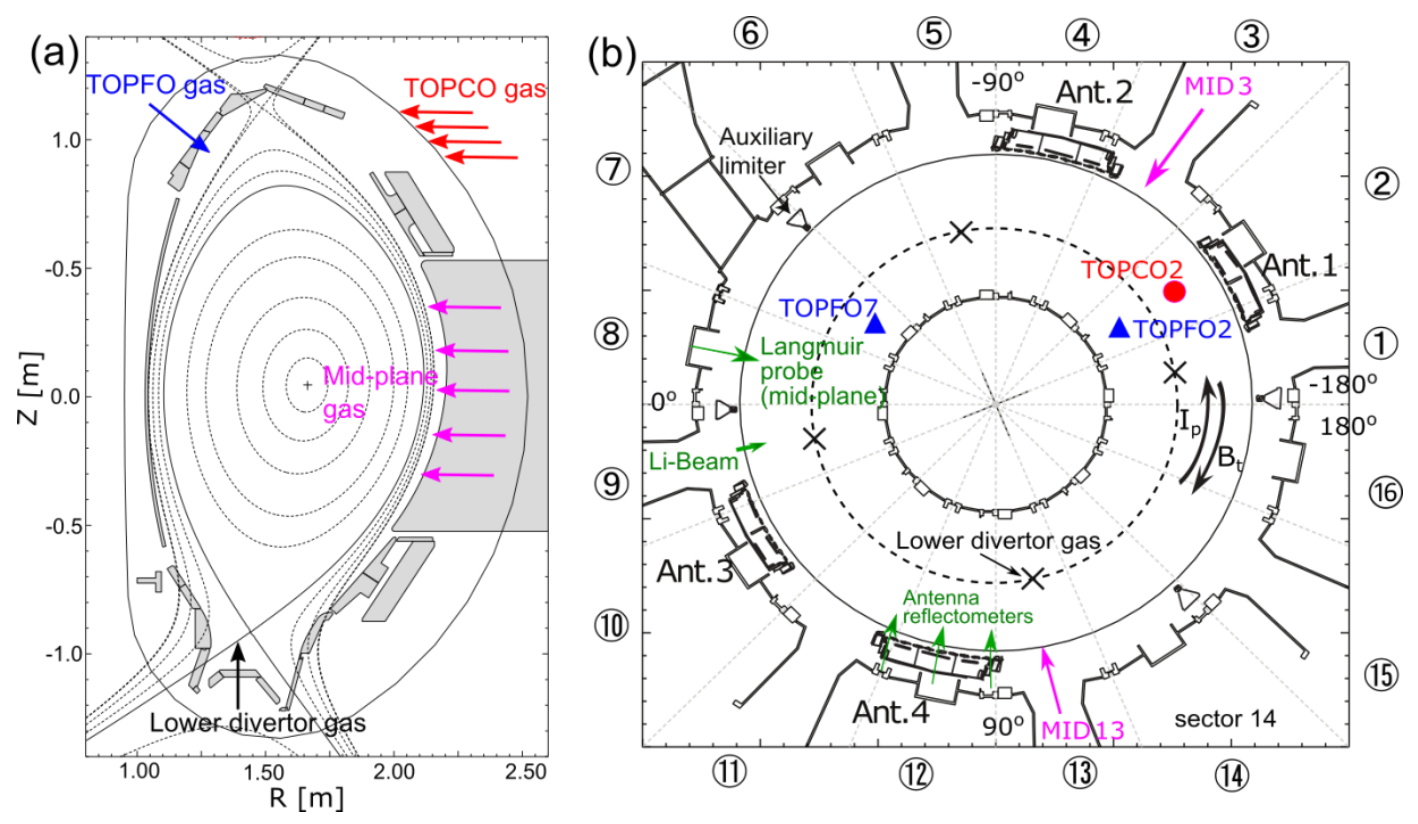

Figure 1. (a) Gas puff locations in the poloidal cross-section; (b) toroidal positions of the gas valves, ICRF antennas and edge density profile measurements. The toroidal angle $-180^{\circ} / 180^{\circ}$ is set at the boundaries of sector 1 and sector 16.
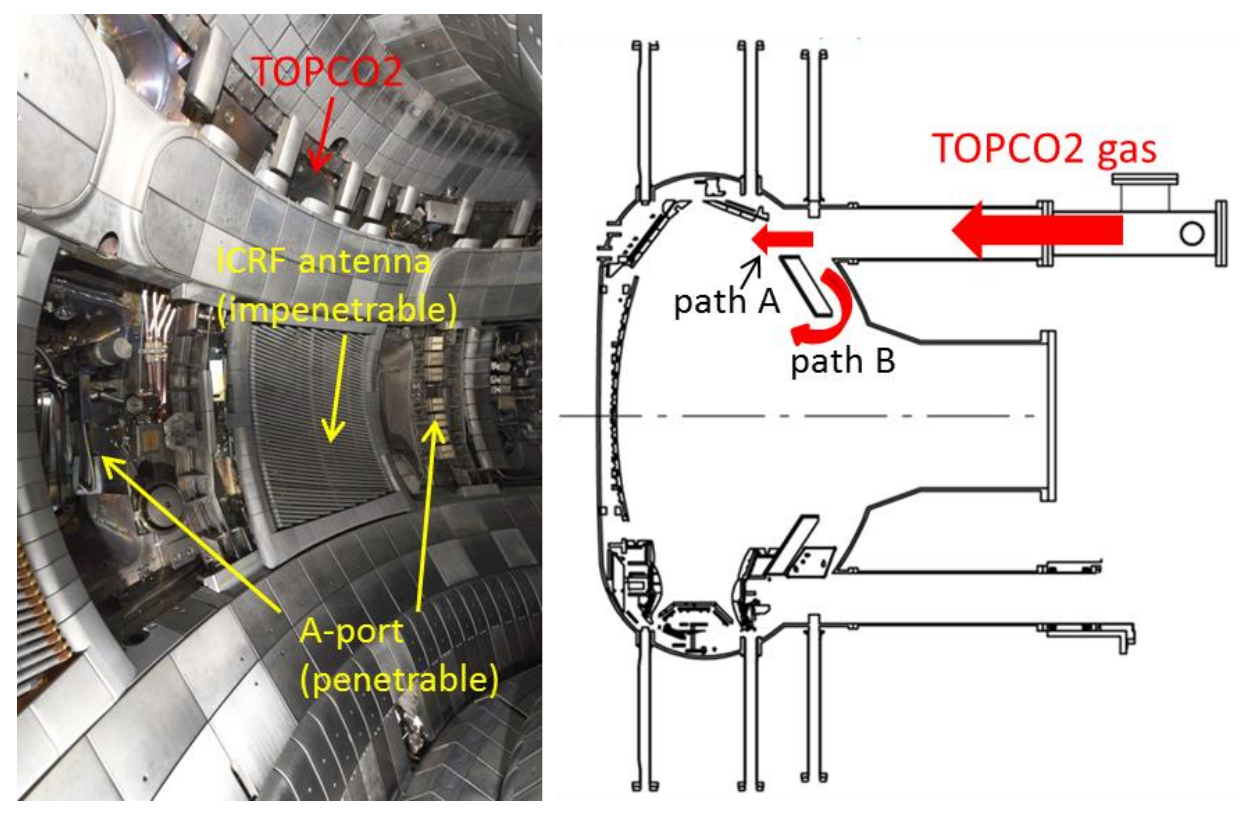

Figure 2. Illustrations of TOPCO2 gas puffing in AUG. For path B, the ICRF antennas are impenetrable to the gas. 

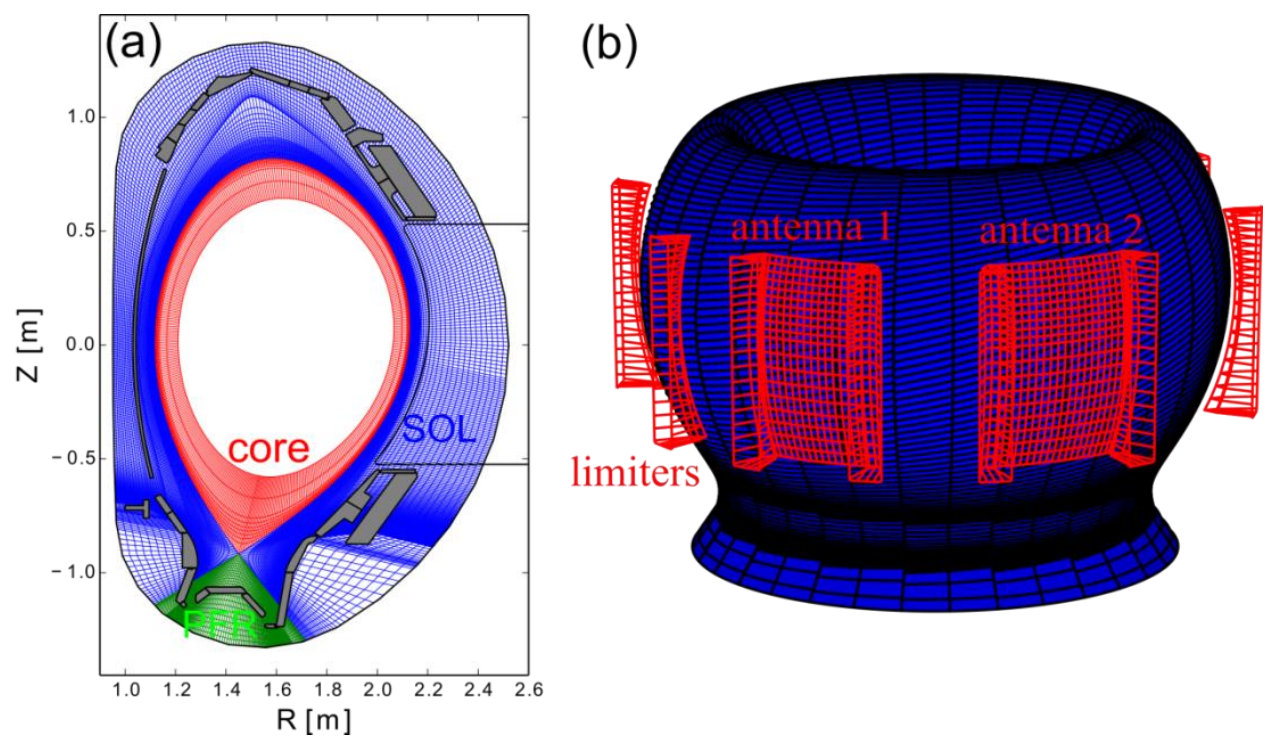

Figure 3. (a) poloidal cross-section of the computational grid including the core, SOL and PFR zones. (b) 3D view of the computational grid, limiters and ICRF antennas.

AUG\#33262
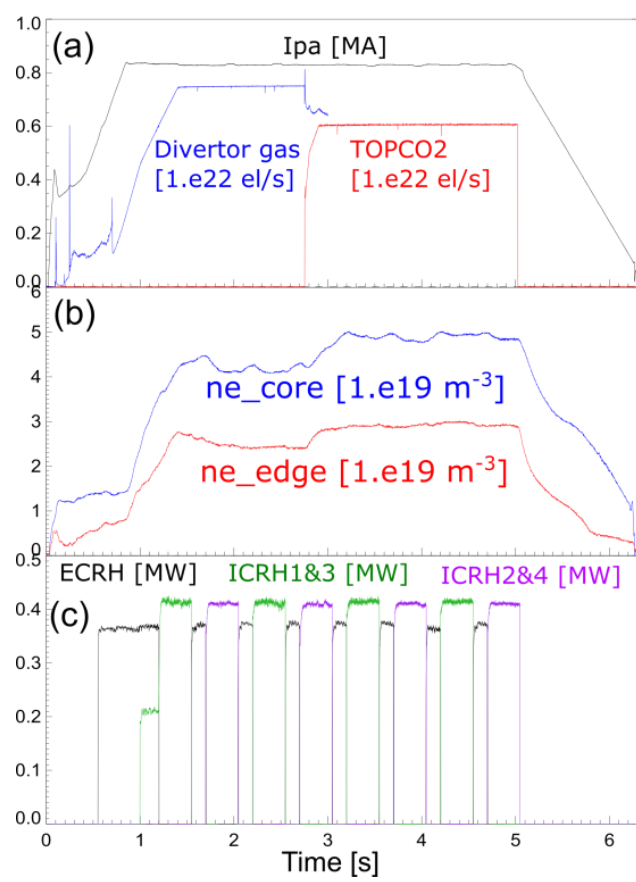

AUG\#33263

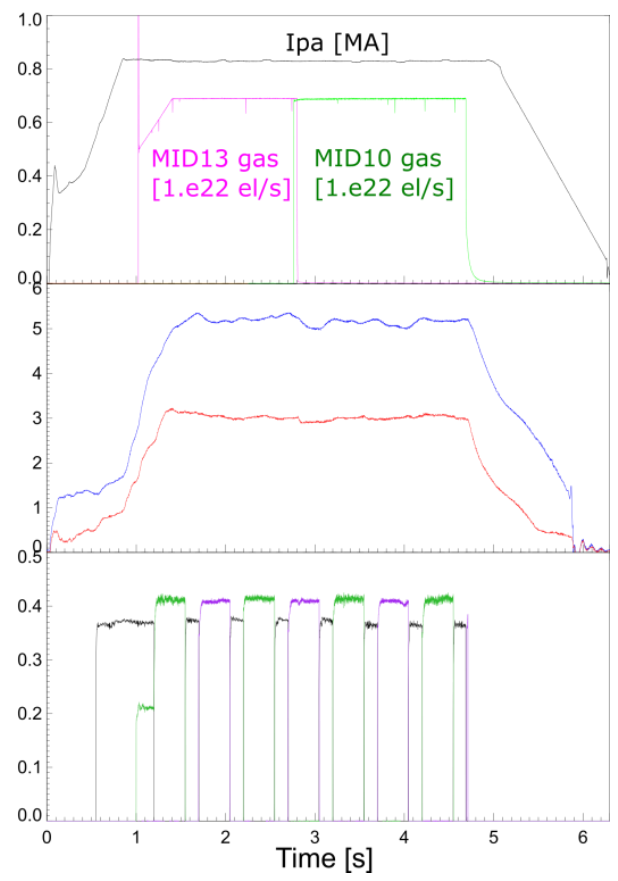

Figure 4. Plasma parameters vs. time for (a) gas puff rate and the plasma current; (b) edge and core line averaged plasma density; (c) heating power. For the ICRF heating, Antennas $1 \& 3$ or Antennas $2 \& 4$ are powered in pairs. 

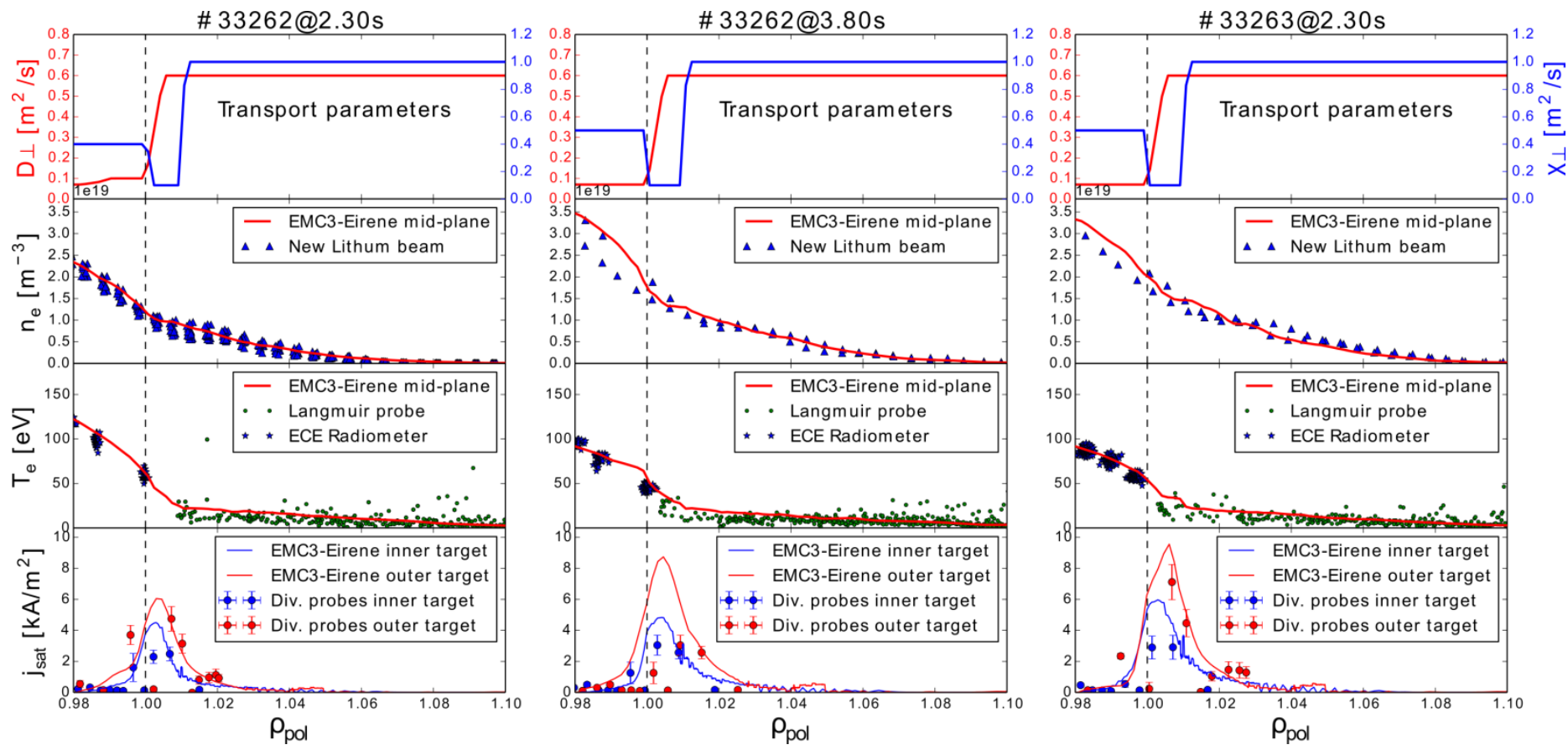

Figure 5. Comparison of simulated profiles with experiment for the DIV (\#33262 at 2.3s), TOPCO2 (\#33262 at 3.8 s) and MID13 (\#33263 at 2.3s) gas puffing cases. From top to bottom: (a) particle and energy transport parameters; (b) mid-plane electron density; (c) mid-plane electron temperature; (d) particle flux to the targets. In the simulations, the input power $\left(\mathrm{P}_{\text {input }}=\mathrm{P}_{\mathrm{ICRF}}+\mathrm{P}_{\mathrm{ECRH}}+\mathrm{P}_{\mathrm{OH}}-\mathrm{P}_{\mathrm{rad}}=0.4 \mathrm{MW}\right)$ and the total gas puff rate $\left(\Phi_{D 2}=6.5 \times 10^{21} \mathrm{el} / \mathrm{s}\right)$ are set as the same for all the cases. The separatrix densities for the DIV, the TOPCO2 and the MID13 gas puffing cases are set as $1.6 \times 10^{19} \mathrm{~m}^{-3}, 2.4 \times 10^{19} \mathrm{~m}^{-3}$ and $2.6 \times 10^{19} \mathrm{~m}^{-3}$, respectively. 

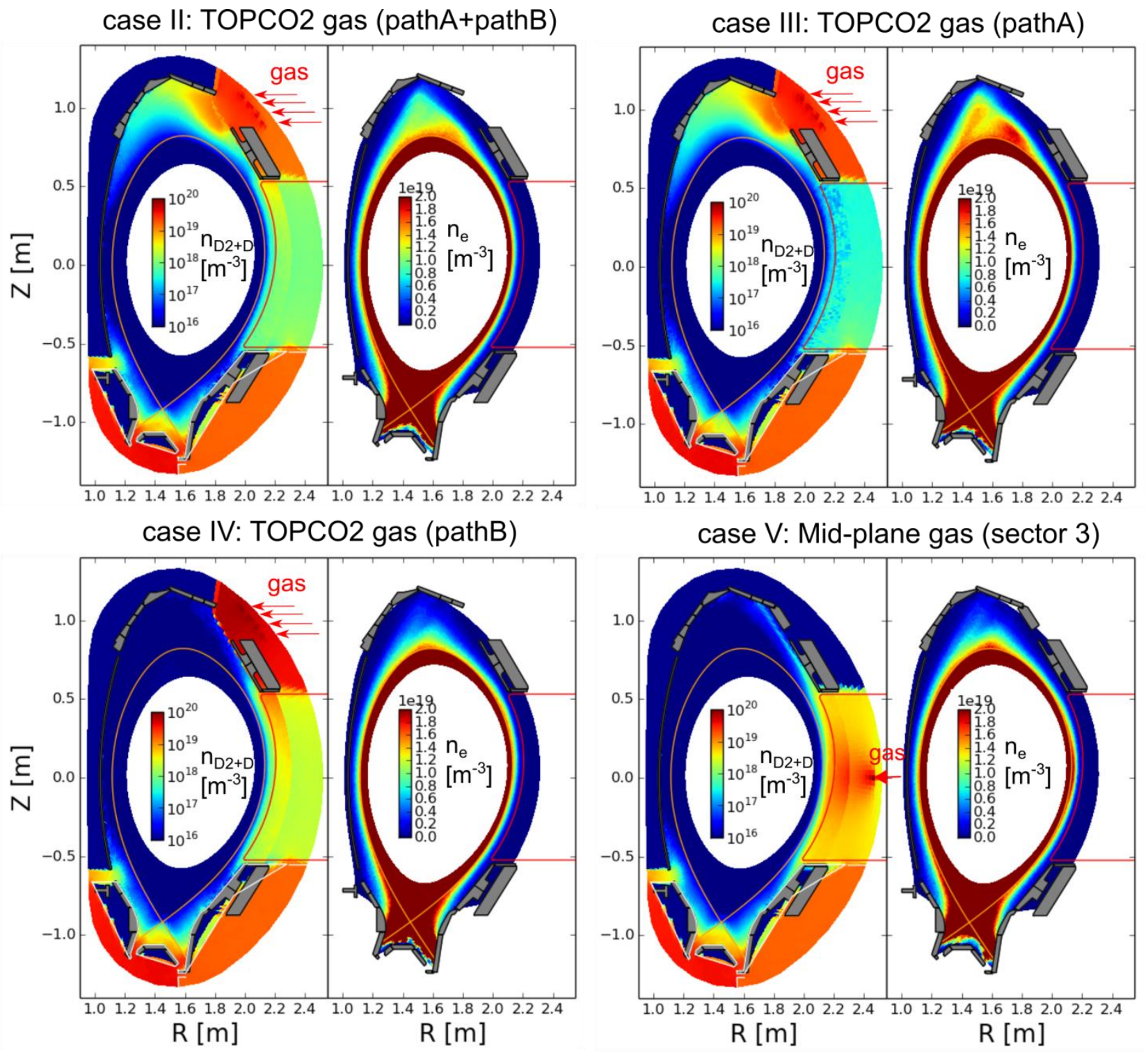

Figure 6. The neutral density $\left(n_{D 2}+n_{D}\right)$ and electron density $\left(n_{e}\right)$ during TOPCO2 and MID13 gas puffing, respectively. For Cases II to IV, the same TOPCO2 gas is used while the gas paths are chosen differently. The plots are made for toroidal angles of $\Phi=-136.4^{\circ}$ for TOPCO2 gas puffing (Cases II, III and IV) and $\Phi=-123.75^{\circ}$ for MID13 gas puffing (Case V), respectively. 


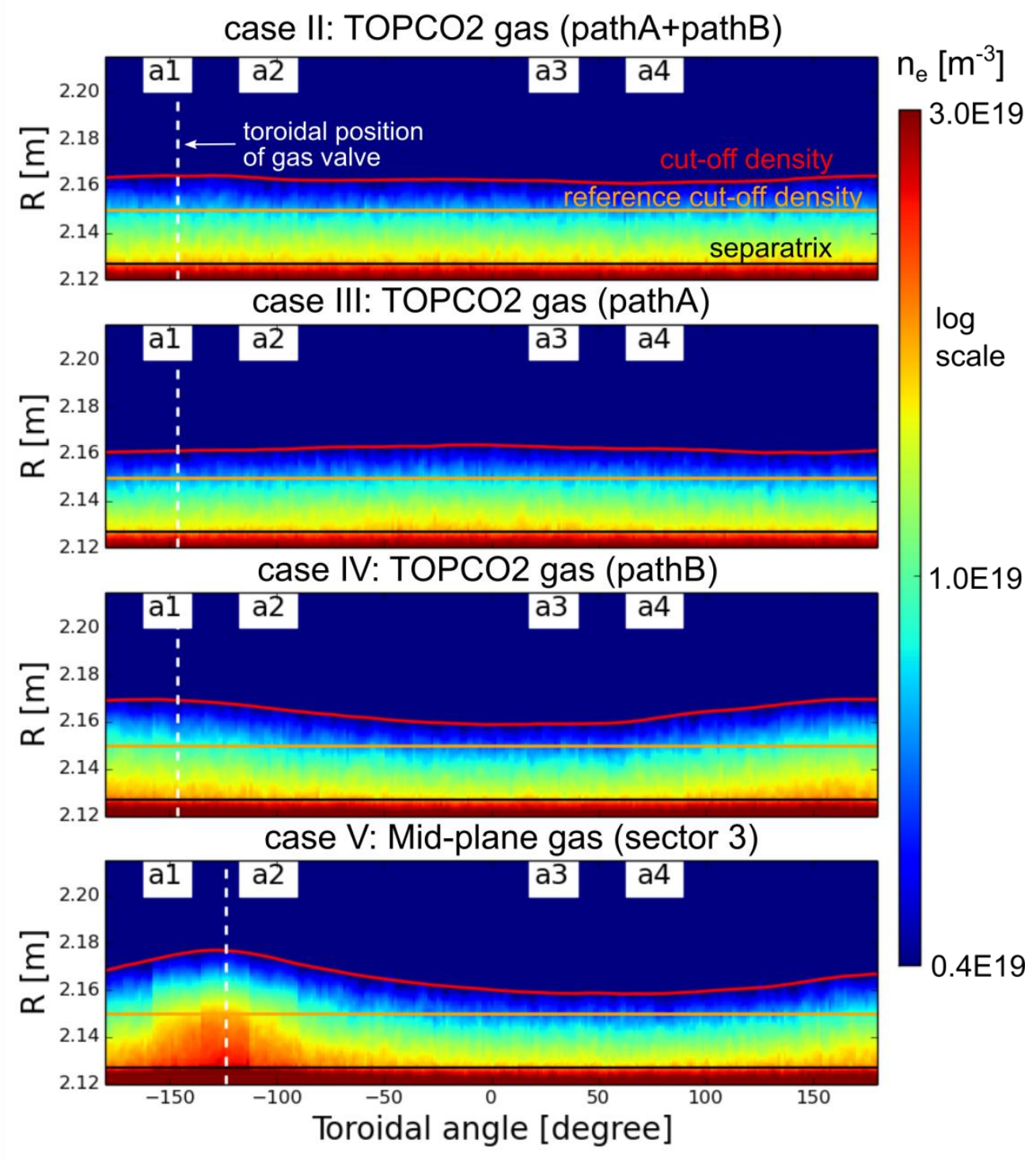

Figure 7. Toroidal cross-sections of the mid-plane SOL electron density $(Z=0.0 \mathrm{~m})$ for top and mid-plane gas puffing (the 4 cases in Figure 6 ). The red line represents the position of cut-off density in each case, with the orange line representing the position of the ICRF cut-off density during DIV gas puffing used as a reference. The vertical dashed line is the toroidal position of the gas valve. a1 to a4 indicate the toroidal locations of the four ICRF antennas. 


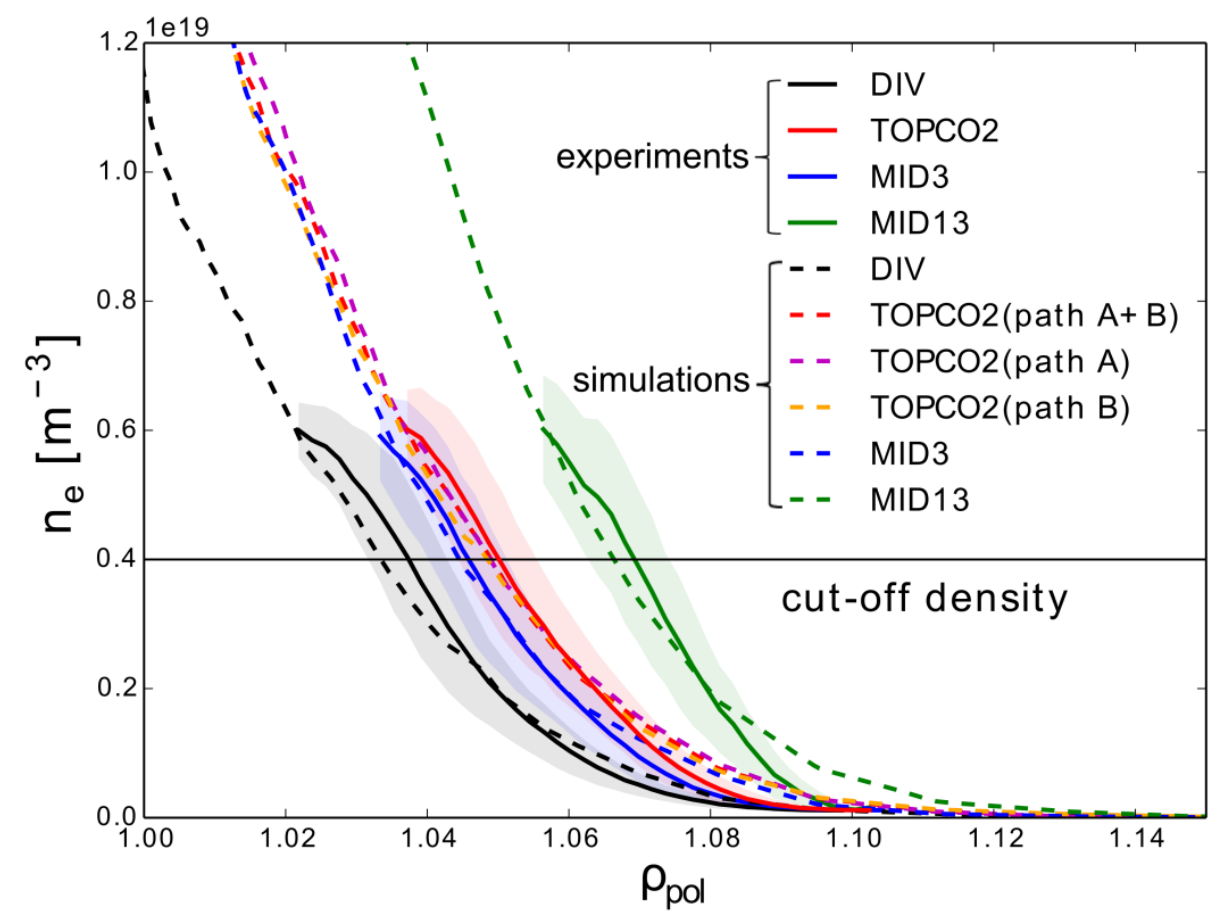

Figure 8. Comparisons of simulated electron density with antenna reflectometer measurements for DIV gas puffing, TOPCO2 gas puffing (Case II), MID3 and MID13 gas puffing.

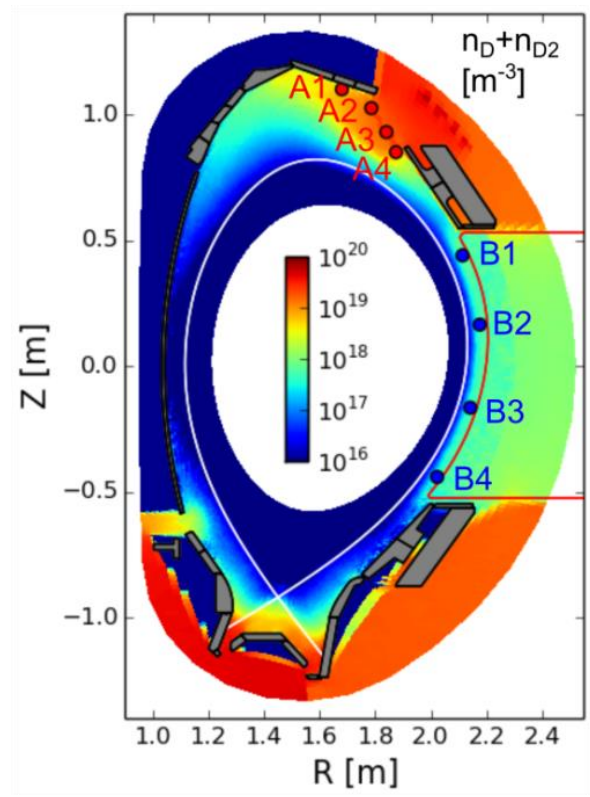

Field line tracings

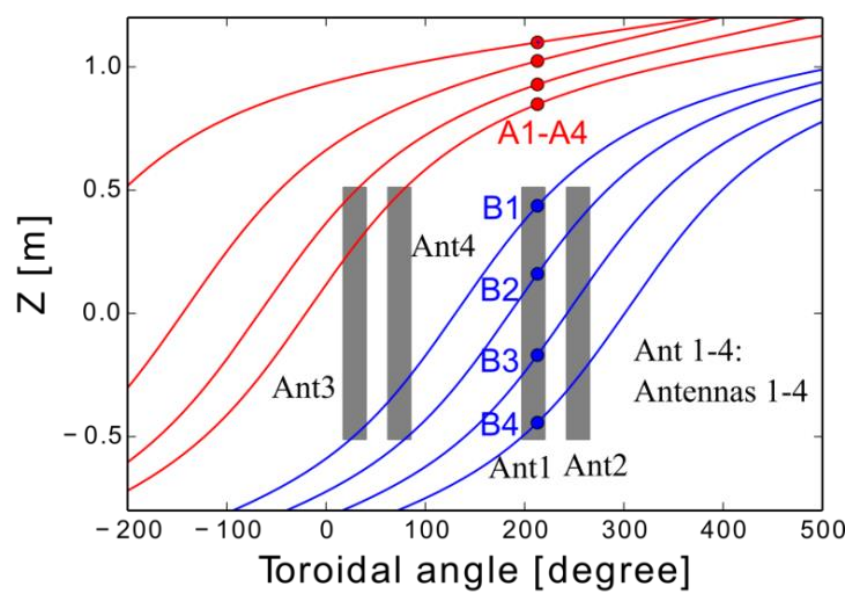

Figure 9. Field line tracing from points in the top and mid-plane gas clouds generated during simulated TOPCO2 gas puffing (Paths $A+B)$. 

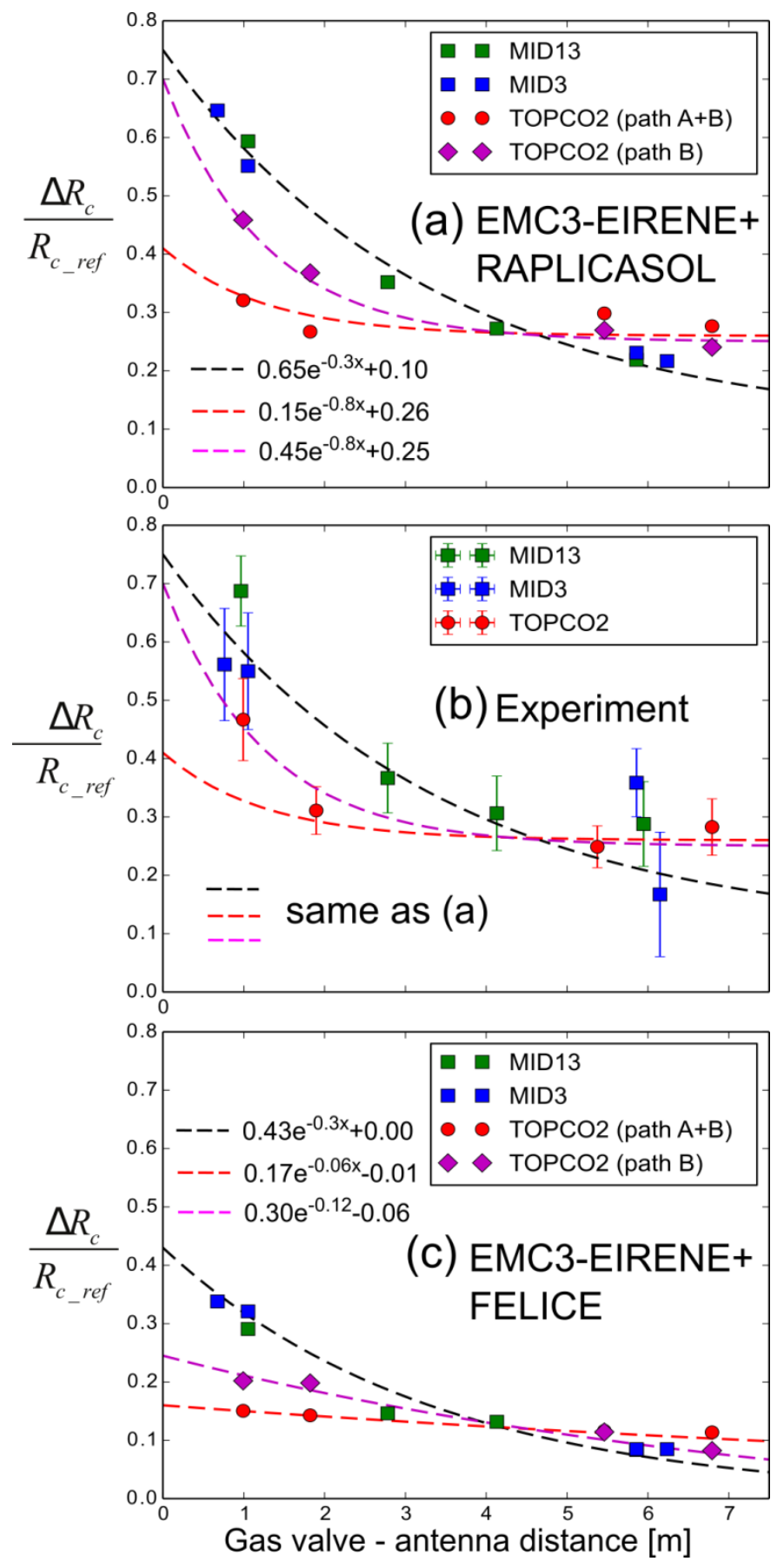

Figure 10. Comparison of experimental and simulated Relative Change of Coupling Resistance (RCCR) values for different mid-plane and TOPCO2 gas puffing cases. (a) RCCR values calculated with the EMC3-EIRENE and RAPLICASOL codes; (b) experimentally measured RCCR values; (c) RCCR values calculated with the EMC3-EIRENE and FELICE codes. The dashed lines are fits to the calculated RCCR values (with exponential functions) in order to facilitate the comparisons between experiments and simulations. The gas valve-antenna distance is the geometrical distance calculated with $\sqrt{h^{2}+d^{2}}$, where $h$ is the vertical distance from the valve to the antenna mid-plane and $d$ is the toroidal distance. 


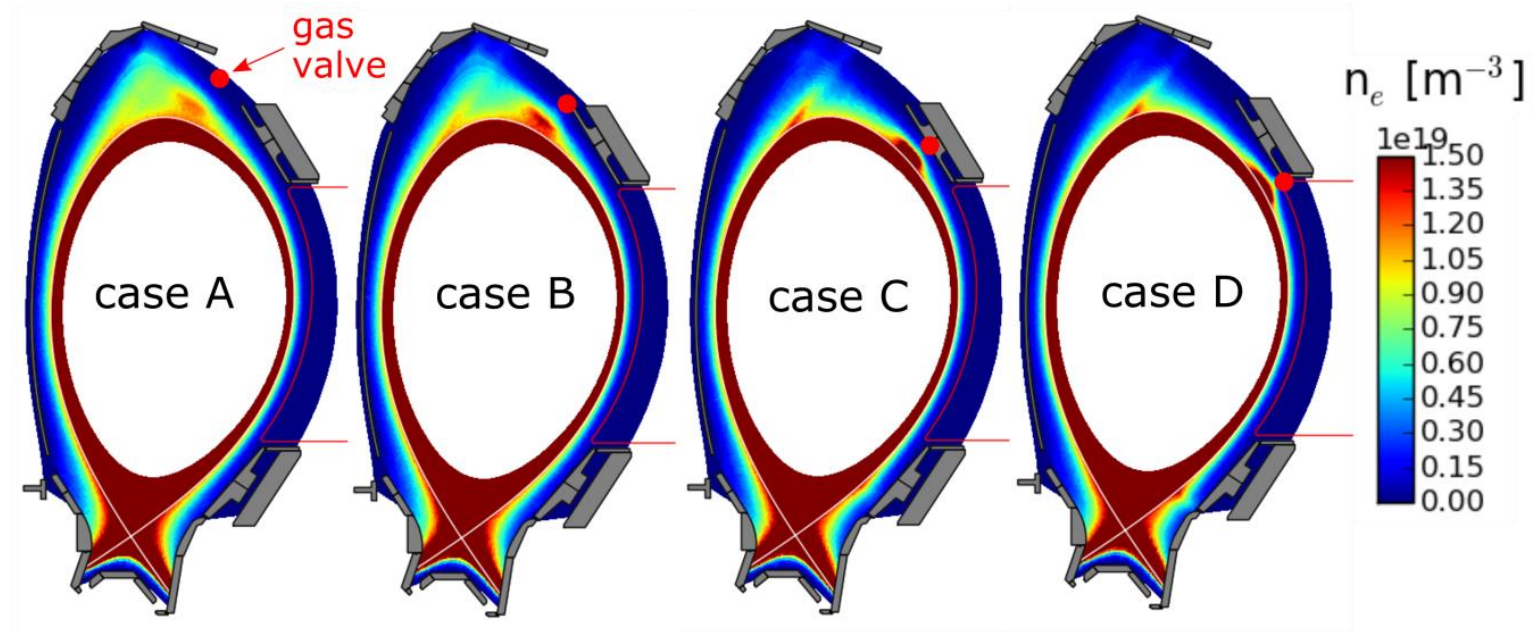

Figure 11. Influence of the outer top gas valve position on the SOL density. The poloidal cross-sections are chosen at $\Phi=\Phi_{\mathrm{v}}=-146.25^{\circ}$ for all four cases.

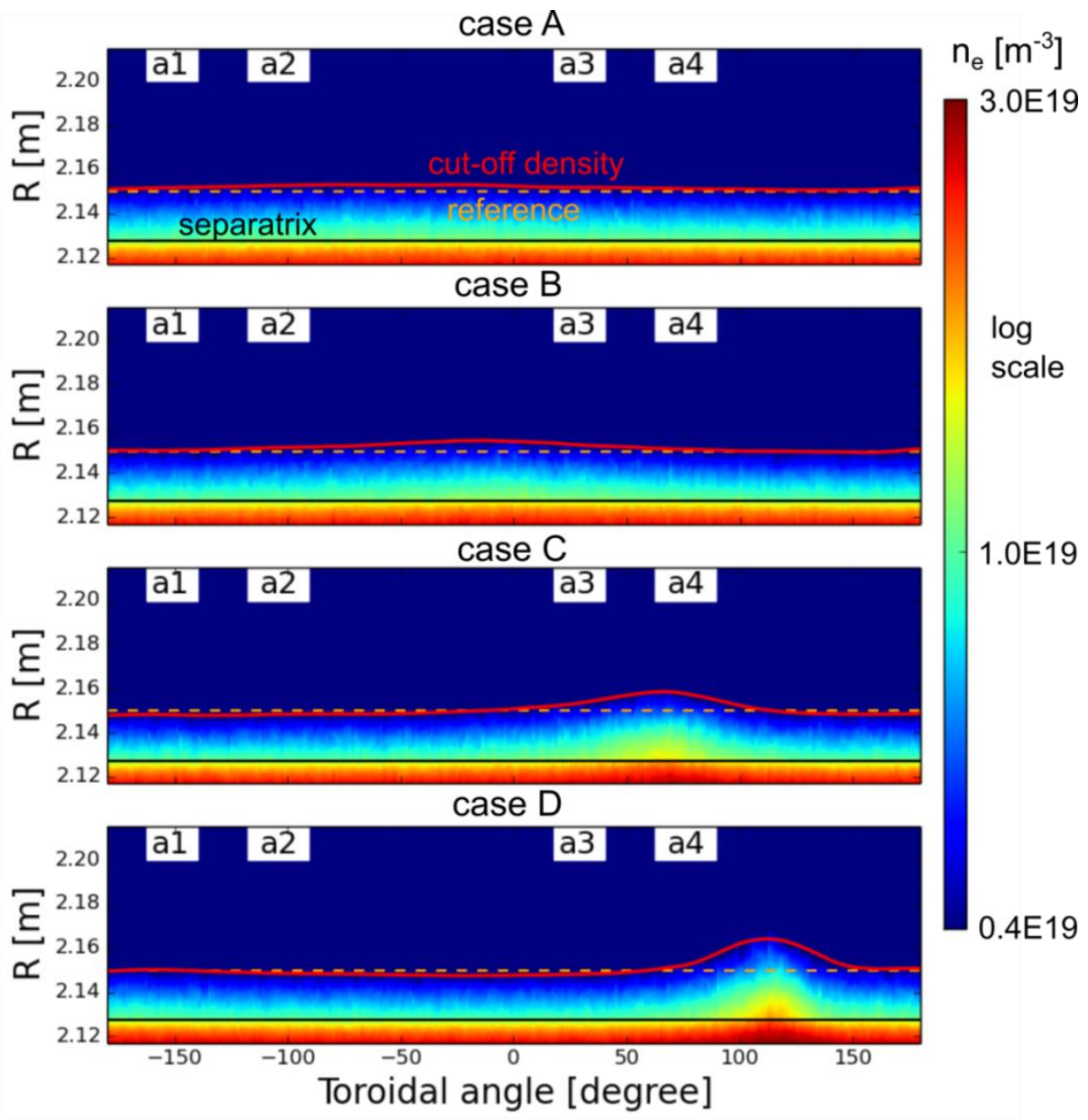

Figure 12. Toroidal cross-sections of the mid-plane electron density $(Z=0.0 \mathrm{~m})$ for the four cases in Figure 11 . The red line represents the position of cut-off density in each case with the dashed orange line giving the position of the cut-off density during DIV gas puffing, used as reference. 